\title{
The Existence of at most Twenty Seven Nonnegative Equilibrium Points in a Class of 3-D Competitive Cubic Systems
}

\author{
Helmar Nunes Moreira \\ Department of Mathematics, University of Brasília, Brasília-DF, CEP70910-900, Brazil
}

\begin{abstract}
This paper presents the stability analysis of equilibrium points of a model involving competition between three species subject to a strong Allee effect which occurs at low population density. By using the software of MAPLE 10, we prove that, under certain conditions, the model has at most twenty seven nonnegative equilibriu m points and, via Lyapunov function, we derive criteria for the asymptotical stability of the unique positive equilibriu m point.
\end{abstract}

Keywords Allee Effect, Competition Model Of Three Species, Lyapunov Theorem

\section{Introduction}

The Allee model of growth has been widely and success fully used as a simple, yet adequate descriptor of the dynamics of small populations or critical depensation model[1], and many theoretical studies (e.g.[2],[3]) have been achieved. The Allee effect refers to reduced fitness or decline in population growth at low population densities. In population models, the Allee effect is often modeled as a threshold, below which there is population extinction.

In the present paper, we consider the Allee effect within the context of the symmetric model of three competing species. We wish to point out that an model of two competing species with Allee effect was proposed and studied in[2-4], and some papers dealing with experiments, simulations, or combinations of these competitive systems among others are described in[5-6]. In Section 2, we introduce the symmetric model of three competing species subject to the Allee effects. The main analytical results on stability analysis of the equilibrium points, are presented in Section 3. Section 4 is devoted to a discussion, in the context of numerical simulation, of the analytical results obtained in this paper. Concluding re marks on the paper are made at the end.

\section{A three-species Competitive System Subject to the Allee Effects}

In the three-species Lotka-Volterra competition models (e.g.[7-11]), it is possible for one- or two species extinction,

* Corresponding author:

helmar@mat.unb.br (helmar nunes moreira)

Published online at http://journal.sapub.org/ajcam

Copyright (C 2012 Scientific \& Academic Publishing. All Rights Reserved or global stability of a positive three-species equilibrium, periodic solutions or a stable heteroclinic orb it (e.g.[12-14]). Here, we shall propose a new three-competitive model that specifically predicts Allee growth of species $x, y$, and $z$, respectively. Keeping this in mind, the model is described as follows:

$$
\begin{gathered}
x^{\prime}=x[(x-a)(1-x)-\alpha y-\beta z]=x f(x, y, z), \\
y^{\prime}=y[(y-b)(1-y)-\beta x-\alpha z]=y g(x, y, z),(2.1) \\
z^{\prime}=z[(z-c)(1-z)-\alpha x-\beta y]=z h(x, y, z), \\
x(0) \geq 0, y(0) \geq 0, z(0) \geq 0,
\end{gathered}
$$

where $x, y$ and $z$ are the population densities, $(x-$ $a 1-x, \quad y-b 1-y$ and $z-c 1-z$ are the quadratic intrinsic growth rates at intermediate densities, $0<a<1,0<b<$ 1 , and $0<c<1$ are the lower threshold of the population densities $x, y$, and $z$, respectively, $\alpha$ and $\beta$ are the coefficients of interspecies competition, and $("=d / d t)$. Throughout this paper we assume that $0<\alpha<$ $1 / 4$ and $0<\beta<1 / 4$.

In our model we consider that the intrinsic growth rates are quadratic and we prove that, under certain conditions, system (2.1) has at most twenty seven nonnegative equilibria. By using the software of MAPLE 10, a numerical example is provided to illustrate the behavior of the system (2.1) for a biologically reasonable range of parameters with only one asymptotically stable equilibrium point and seven unstable equilibrium points in $R_{+}^{3}$. We believe that this is the first time that the three-species competition system (2.1) has been formulated and analyzed in the literature.

\subsection{Boundedness of the Solutions}

Consider the system (2.1). Obviously the functions $f, g$, and $h$ are continuous and Lipschitzian with respect to all independent variables on $R_{+}^{3}=\{(x, y, z) / x \geq 0, y \geq$ $0, z \geq 0\}$. Therefore, a solution of the system (2.1) with 
nonnegative initial conditions exists and is unique. The basic existence and uniqueness theorem for differential equations ensures that

Lemma 2.1. The positive cone Int $R_{+}^{3}$ is invariant for system (2.1).

Lemma 2.2. The solutions $x(t), y(t)$, and $z(t)$ of system (2.1) with positive initial conditions are bounded for all $t \geq 0$.

Proof. Since $x^{\prime} \leq x[(x-a)(1-x)]$, then $\limsup _{t \rightarrow+\infty} x(t) \leq 1$. Here, we consider the case of strong Allee effect :

$$
x^{\prime}=f(x)=x(x-a)(1-x),
$$

where $0<a<1$ is the survival threshold. There are three equilibrium points $x_{o}=0$, and $0<x_{+}^{1}=a<x_{+}^{2}=1$. The relative extre ma of the function $f(x)$ are

$$
x_{*}^{2,1}=\frac{\left[(a+1) \pm \sqrt{a^{2}-a+1}\right]}{3},
$$

which give the points of inflection of the graph of $x$ versus t. The solutions are increasing and concave down when $x_{*}^{2}<x<1$; increasing and concave up when $K_{o}<x<x_{*}^{2}$; decreasing and concave down when $x_{*}^{1}<x<K_{o}$; decreasing and concave up when $0=x_{o}<$ $x<x_{*}^{1}$ or $x>1$. We conclude that $x_{o}=0$ and $x_{+}^{2}=1$ are sinks; and $x_{+}^{1}=a$ is a source. Then if the initial population size is below $a$, the population $x=x(t)$ will die out.

Similarly to $y$ and $z$, respectively.

\section{Existence and Stability of Equilibrium Points}

Computations of the boundary equilibria and the analysis of the existence of positive equilibrium points and their stability for system (2.1), provide the information needed to determine the coexistence or extinction of species. To do so, we compute the Jacobian matrix $J(E)$ of (2.1). The signs of the real parts of the eigenvalues of $J(E)$ evaluated at a given equilibrium point $E=(x, y, z)$ determine its stability. Here

$$
J(E)=\left[\begin{array}{ccc}
(a+1-2 x) x+f & -\alpha x & -\beta x \\
-\beta y & (b+1-2 y) y+g & -\alpha y \\
-\alpha z & -\beta z & (c+1-2 z) z+h
\end{array}\right]
$$

where $f, g$, and $h$ are as in (2.1), $a<1, b<1, c<1$, $\alpha<\frac{1}{4}$ and $\beta<\frac{1}{4}$, and all the parameters are positive. System (2.1) has at most twenty seven non-negative equilibria:

$E_{000}=(0,0,0)$, with eigenvalues $J\left(E_{000}\right)=\{-a<$ $0,-b<0,-c<0\}$. Thus, $E_{000}$ is locally asymptotically stable;

$E_{a 00}=(a, 0,0)$, with eigenvalues $J\left(E_{a 00}\right)=\{-b-$ $\beta a<0,-c-\alpha a<0, a(1-a)>0\}$. This implies that $E_{a 00}$ is unstable;

$E_{100}=(1,0,0)$, where eigenvalues $J\left(E_{100}\right)=\{a-1<$ $0,-b-\beta<0,-c-\alpha<0\}$. Thus, $E_{100}$ is locally asymptotically stable;

$E_{0 b 0}=(0, b, 0)$, where eigenvalues $J\left(E_{0 b 0}\right)=\{b(1-$ b) $>0,-a-\alpha b<0,-c-\beta b<0\}$. This implies that
$E_{0 b 0}$ is unstable;

$E_{010}=(0,1,0)$, with eigenvalues $J\left(E_{010}\right)=\{-a-\alpha<$ $0, b-1<0,-\mathrm{c}-\beta<0\}$. Thus, $E_{010}$ is locally asymptotically stable;

$E_{00 c}=(0,0, c)$, where eigenvalues $J\left(E_{00 c}\right)=\{c(1-$ c) $>0,-a-\beta c<0,-b-\alpha c<0\}$. This implies that $E_{00 c}$ is unstable;

$E_{001}=(0,0,1)$, with eigenvalues $J\left(E_{001}\right)=\{-a-\beta<$ $0,-b-\alpha<0, \mathrm{c}-1<0\}$. Thus , $E_{001}$ is locally as ymptotically stable;

Now we establish criteria for the existence and stability of the equilibrium $E_{++0}=\left(x^{*}, y^{*}, 0\right)$. For this case, one of the three competitors goes to extinction depending on the initial values and the coexistence of three competing species described by (2.1) is not possible. Thus the exclusion principle holds [10].

When system (2.1) is restricted to $R_{x y}^{2}$, we obtain the following subsystem:

$$
\begin{gathered}
x^{\prime}=x[(x-a)(1-x)-\alpha y]=x f(x, y, 0), \\
y^{\prime}=y[(y-b)(1-y)-\beta x]=y g(x, y, 0) .
\end{gathered}
$$

An interior planar equilibrium $E_{++0}=\left(x^{*}, y^{*}, 0\right)$ occurring in the $x-y$ plane exists if and only if the algebraic system $f\left(x^{*}, y^{*}, 0\right)=0, g\left(x^{*}, y^{*}, 0\right)=0$ has a positive solution. A routine computation yields

and $p\left(x^{*}\right)=0$, where

$$
y^{*}=\frac{\left(x^{*}-a\right)\left(1-x^{*}\right)}{\alpha}
$$

$$
\begin{gathered}
p(x)=\lambda_{14} x^{4}+\lambda_{13} x^{3}+\lambda_{12} x^{2}+\lambda_{11} x+\lambda_{10}, \\
\lambda_{14}=\frac{1}{\alpha^{2}}>0, \lambda_{13}=-\frac{2(a+1)}{\alpha^{2}}<0, \\
\lambda_{12}=\frac{\left[(a+1)^{2}+2 a+\alpha(b+1)\right]}{\alpha^{2}}>0, \\
\lambda_{11}=\frac{\left[\alpha^{2} \beta-\alpha(a+1)(b+1)-2 a(a+1)\right]}{\alpha^{2}}<0, \\
\lambda_{10}=\frac{\left[\alpha^{2} b+a^{2}+\alpha a(b+1)\right]}{\alpha^{2}}>0
\end{gathered}
$$

Using the rule of signs of Descartes it follows: (a) There are four sign changes in $p(x)$, so there are 4,2 or 0 positive roots; (b) There are no sign changes at $p(-x)$, so there are no negative roots. Hence, at most four positive equilibrium points are possible in the $x-y$ plane[15].

Under certain conditions on the parameters we have the following geometric interpretation (see Fig.3.1):

Proposition 3.1. Let $E_{++0}=\left(x^{*}, y^{*}, 0\right)$ denote an interior equilibrium in the $x-y$ plane. Then $E_{++0}$ is the intersection of ellipses $E_{1}$ and $E_{2}$ defined by

$$
\begin{gathered}
E_{1}: x=A+B \cos (t), y=C \sin (t), \\
E_{2}: x=E \cos (t), y=D+F \sin (t),-\pi \leq t \leq \pi .
\end{gathered}
$$
where

$$
\begin{gathered}
\Delta=(a+1)(b+1)-\alpha \beta>0, A=\frac{\Delta}{2(b+1)}, D=\frac{\Delta}{2(a+1)}, \\
B^{2}=\frac{\Delta^{2}-4(b+1)[a(b+1)+\alpha b]}{4(b+1)^{2}}, C^{2}=\left(\frac{b+1}{\alpha}\right) B^{2}, \\
F^{2}=\frac{\Delta^{2}-4(a+1)[b(a+1)+\beta a]}{4(a+1)^{2}}, E^{2}=\left(\frac{a+1}{\beta}\right) F^{2}
\end{gathered}
$$


Proposition 3.2. Consider the system (2.1). Suppose that there are four interior equilibria $E_{++0}^{i}=\left(x^{*}, y^{*}, 0\right), i=$ $1,2,3,4$, in the $x-y$ plane; that is, $\mathrm{A}, \mathrm{B}, \mathrm{C}$ and $\mathrm{D}$, respectively. Then only one equilibrium $\mathrm{C}$ of coexisting populations is locally stable and its basin of attraction is bounded by the stable separatrices of the saddles $B$ and $D$, both coming from the unstable node $A$.
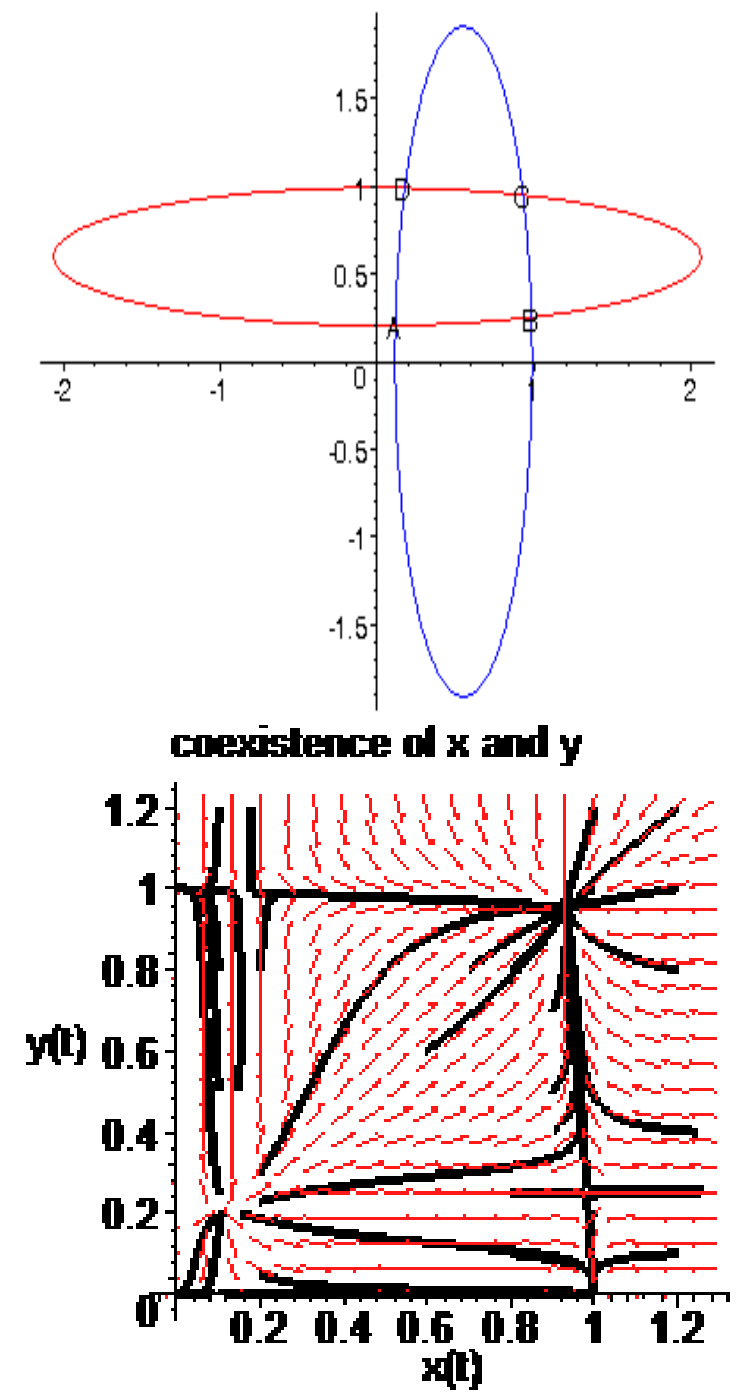

Figure 3.1. Intersection between two ellipses $E_{1}, E_{2}$. The equilibrium points are: $A=E_{++0}^{1}$ is an unstable node; $B=E_{++0}^{2}$ is a saddle point; $C=E_{++0}^{3}$ is locally asymptotically stable; $D=E_{++0}^{4}$ is a saddle point. Here $a=$ $0.1, b=0.2, c=0.15, \alpha=\frac{1}{16}, \beta=\frac{1}{25}$ (see Section 4)

Similar results for the existence and stability of the equilibrium points $E_{+0+}=\left(x^{*}, 0, z^{*}\right)$ and $E_{0++}=$ $\left(0, y^{*}, z^{*}\right)$ are obtained from $(2.1)$.

\subsection{Existence, Stability and Linearization of Positive \\ Equilibrium Points}

Let $E=\left(x^{*}, y^{*}, z^{*}\right)$ denote an interior equilibrium point of $R_{+}^{3}$, if it exists. It follows from direct substitution and algebraic manipulation:

Proposition 3.3. System (2.1) has at most eight equilibrium points in the interior of $R_{+}^{3}$. Their equilibrium values $x^{*}, y^{*}$ and $z^{*}$ are given by

$$
\begin{aligned}
& x^{*}=A_{1}+A_{2} \sqrt{\Delta_{1}}, \\
& z^{*}=B_{1}+B_{2} \sqrt{\Delta_{2}},
\end{aligned}
$$

and $y^{*}$ is a positive root of

$$
F(y)=D_{8} y^{8}+D_{7} y^{7}+D_{6} y^{6}+D_{5} y^{5}+D_{4} y^{4}+D_{3} y^{3}+D_{2} y^{2}+D_{1} y+D_{0}
$$

where

$$
\begin{aligned}
& D_{8}=-P_{1}^{4}<0, D_{7}=-4 P_{2} P_{1}^{3}>0, \\
& D_{6}=2 M_{1} C_{1}^{2} P_{1}^{2}+2 N_{1} C_{2}^{2} P_{1}^{2}-\left(4 P_{3} P_{1}^{3}+6 P_{1}^{2} P_{2}^{2}\right), \\
& D_{5}=2 C_{1}^{2}\left(2 M_{1} P_{1} P_{2}+M_{2} P_{1}^{2}\right)+2 C_{2}^{2}\left(2 N_{1} P_{1} P_{2}+N_{2} P_{1}^{2}\right)-\left(4 P_{1} P_{2}^{3}+12 P_{2} P_{3} P_{1}^{2}\right) \\
& D_{4}=2 M_{1} N_{1} C_{1}^{2} C_{2}^{2}-M_{1}^{2} C_{1}^{4}-N_{1}^{2} C_{2}^{4}+ \\
& 2 C_{1}^{2}\left(M_{1} P_{2}^{2}+2 M_{1} P_{1} P_{3}+2 M_{2} P_{1} P_{2}+M_{3} P_{1}^{2}\right)+ \\
& 2 C_{2}^{2}\left(N_{1} P_{2}^{2}+2 N_{1} P_{1} P_{3}+2 N_{2} P_{1} P_{2}+N_{3} P_{1}^{2}\right)- \\
& \left(6 P_{1}^{2} P_{3}^{2}+12 P_{1} P_{3} P_{2}^{2}+P_{2}^{4}\right) \\
& D_{3}=2 C_{1}^{2} C_{2}^{2}\left(M_{1} N_{2}+M_{2} N_{1}\right) \\
& -2 M_{1} M_{2} C_{1}^{4}-2 N_{1} N_{2} C_{2}^{4}+ \\
& 2 C_{1}^{2}\left(2 M_{1} P_{2} P_{3}+M_{2} P_{2}^{2}+2 M_{2} P_{1} P_{3}+2 M_{3} P_{1} P_{2}\right)+ \\
& 2 C_{2}^{2}\left(2 N_{1} P_{2} P_{3}+N_{2} P_{2}^{2}+2 N_{2} P_{1} P_{3}+2 N_{3} P_{1} P_{2}\right)- \\
& \left(4 P_{3} P_{2}^{3}+12 P_{1} P_{2} P_{3}^{2}\right) \text {, } \\
& D_{2}=2 C_{1}^{2} C_{2}^{2}\left(M_{1} N_{3}+M_{2} N_{2}+M_{3} N_{1}\right)- \\
& C_{1}^{4}\left(M_{2}^{2}+2 M_{1} M_{3}\right)-C_{2}^{4}\left(N_{2}^{2}+2 N_{1} N_{3}\right)+ \\
& 2 C_{1}^{2}\left(M_{1} P_{3}^{2}+2 M_{2} P_{2} P_{3}+M_{3} P_{2}^{2}+2 M_{3} P_{1} P_{3}\right)+ \\
& 2 C_{2}^{2}\left(N_{1} P_{3}^{2}+2 N_{2} P_{2} P_{3}+N_{3} P_{2}^{2}+2 N_{3} P_{1} P_{3}\right)- \\
& \left(6 P_{2}^{2} P_{3}^{2}+4 P_{1} P_{3}^{3}\right) \\
& D_{1}=2 C_{1}^{2} C_{2}^{2}\left(M_{2} N_{3}+M_{3} N_{2}\right)- \\
& 2 M_{2} M_{3} C_{1}^{4}-2 N_{2} N_{3} C_{2}^{4}+ \\
& 2 C_{1}^{2}\left(M_{2} P_{3}^{2}+2 M_{3} P_{2} P_{3}\right)+ \\
& 2 C_{2}^{2}\left(N_{2} P_{3}^{2}+2 N_{3} P_{2} P_{3}\right)-4 P_{2} P_{3}^{3},
\end{aligned}
$$

$D_{0}=P_{3}^{4}+2 M_{3} N_{3} C_{1}^{2} C_{2}^{2}-\left(P_{3}^{2}-M_{3} C_{1}^{2}\right)^{2}-\left(P_{3}^{2}-N_{3} C_{2}^{2}\right)^{2}$,

$A_{1}=\frac{a+1}{2}+\frac{\beta^{2}}{2 \alpha}, A_{2}=\frac{1}{2 \alpha}, B_{1}=\frac{c+1}{2}+\frac{\alpha^{2}}{2 \beta}, B_{2}=\frac{1}{2 \beta}$,

$$
\Delta_{1}=M_{1} y^{2}+M_{2} y+M_{3}
$$$$
\Delta_{2}=N_{1} y^{2}+N_{2} y+N_{3} \text {, }
$$

$M_{1}=N_{1}=4 \alpha \beta>0, M_{2}=-4 \alpha\left[(b+1) \beta+\alpha^{2}\right]<0$,

$$
\begin{gathered}
M_{3}=\left[(a+1) \alpha+\beta^{2}\right]^{2}+4 \alpha(b \beta-a \alpha) \\
=\beta^{4}+2(a+1) \alpha \beta^{2}+4 b \alpha \beta+(a-1)^{2} \alpha^{2}>0, \\
N_{2}=-4 \beta\left[(b+1) \alpha+\beta^{2}\right]<0, \\
N_{3}=\left[(c+1) \beta+\alpha^{2}\right]^{2}+4 \beta(b \alpha-c \beta) \\
=\alpha^{4}+2(c+1) \beta \alpha^{2}+4 b \alpha \beta+(c-1)^{2} \beta^{2}>0,
\end{gathered}
$$




$$
\begin{gathered}
C_{1}=(a+1) A_{2}-2 A_{1} A_{2}=-\frac{\beta^{2}}{\alpha^{2}}<0, C_{2}=-\beta B_{2}=-\frac{1}{2}, \\
P_{1}=M_{1} A_{2}^{2}=\frac{\beta}{\alpha}>0, P_{2}=\alpha+M_{2} A_{2}^{2}=-\frac{(b+1) \beta}{\alpha}<0, \\
P_{3}=A_{1}^{2}-(a+1) A_{1}+M_{3} A_{2}^{2}+\beta B_{1}+a \\
=\frac{\beta^{4}}{2 \alpha^{2}}+\frac{(a+1) \beta^{2}}{2 \alpha}+\left(\frac{b}{\alpha}+\frac{c+1}{2}\right) \beta+\frac{\alpha^{2}}{2}>0 .
\end{gathered}
$$

Corollary 3.1. Suppose that $D_{6}<0, D_{5}>0, D_{4}<$ $0, D_{3}>0, D_{2}<0, D_{1}>0$, and $D_{0}<0$. Then $F(y)$ has 8,6 , 4,2 or 0 positive roots.

Corollary 3.2. Suppose $D_{0}>0$. Then $F(y)$ has at least one positive root.

Proof. Clearly, $F(0)=D_{0}>0$, and $\lim _{y \rightarrow \infty} F(y)=$ $-\infty$. Hence, there exists a $y^{*} \in(0, \infty)$ so that $F\left(y^{*}\right)=0$. This completes the proof.

Remark 3.1 Using the software MAPLE, we obtain the following numerical examples:(i) $a=0.5, b=0.25, c=$ $0.2, \alpha=\frac{1}{16}, \beta=\frac{1}{9}=>$ none positive equilibrium; (ii) $a=0.4, b=0.25, c=0.3, \alpha=\frac{1}{16}, \beta=\frac{1}{9}=>$ two positive equilibriums with eigenvalues $(-,+,+),(+,+,+)$; (iii) $a=$ $0.4, b=0.25, c=0.2, \alpha=\frac{1}{16}, \beta=\frac{1}{9}=>\quad$ two positive equilibriums with eigenvalues $(-,+,+),(+, \alpha \pm i \beta), \alpha>0$; (iv) $a=0.4, b=0.25, c=0.1, \alpha=\frac{1}{16}, \beta=\frac{1}{9}=>\quad$ four positive equilibriums with eigenvalues $(+,+,+),(+,-,+),(-,-$ $,+),(-,+,+)$.

It is always informative to draw the set of positive equilibrium points of the system (2.1) in $R_{+}^{3}$. Here the set is defined by the intersection of the surfaces:

$$
f(x, y, z)=0, g(x, y, z)=0, h(x, y, z)=0
$$

Under certain conditions on the parameters of the system (2.1), we obtain (see Fig 3.2):

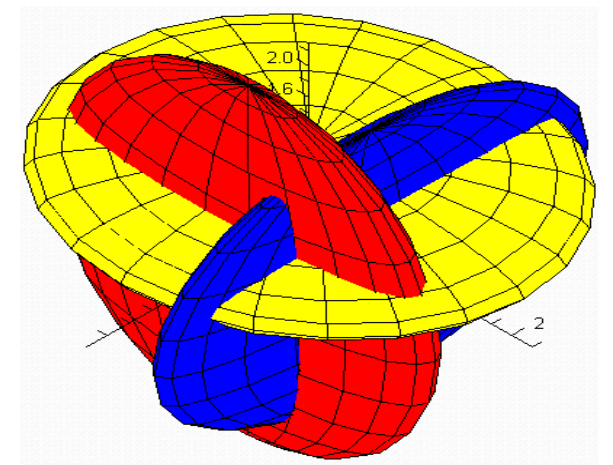

Figure 3.2. Intersection between ellipsoids $S 1, S 2$, and $S 3$. There are 8 equilibrium points in $R_{+}^{3}$. Here $a=0.1, b=0.2, c=0.15, \alpha=\frac{1}{16}, \beta=\frac{1}{25}$.

Proposition 3.4. Let $E=\left(x^{*}, y^{*}, z^{*}\right)$ denote the interior equilibrium of the system (2.1), if it exists. Then $E$ is the intersection of three ellipsoids $S_{1}, S_{2}$ and $S_{3}$ :
$S 1: x=\Delta_{11}+\nabla_{11} \sin \sin (s) \cos \cos (t), y$

$=\nabla_{12} \sin \sin (s) \sin \sin (t), z$

$=\nabla_{13} \cos \cos (s)$,

$S 2: x=\nabla_{21} \sin \sin (s) \cos \cos (t), y$

$=\Delta_{22}+\nabla_{22} \sin \sin (s) \sin \sin (t), z$

$=\nabla_{23} \cos \cos (s)$,

$S 3: x=\nabla_{31} \sin \sin (s) \cos \cos (t), y$

$=\nabla_{32} \sin \sin (s) \sin \sin (t), z$

$=\Delta_{33}+\nabla_{33} \cos \cos (s)$,

$$
0 \leq s \leq \pi, 0 \leq t \leq 2 \pi
$$

$$
\Delta^{2}-4 A_{i i} \nabla_{i}>0 ; i=1,2,3 .
$$

where

$$
\begin{aligned}
& \Delta_{11}=\frac{\Delta}{2 A_{11}}, \nabla_{11}^{2}=\frac{\Delta^{2}-4 A_{11} \nabla_{1}}{4 A_{11}^{2}}, \\
& \nabla_{12}^{2}=\frac{\Delta^{2}-4 A_{11} \nabla_{1}}{4 A_{11} A_{12}}, \nabla_{13}^{2}=\frac{\Delta^{2}-4 A_{11} \nabla_{1}}{4 A_{11} A_{13}} \text {, } \\
& \Delta_{22}=\frac{\Delta}{2 A_{22}}, \nabla_{21}^{2}=\frac{\Delta^{2}-4 A_{22} \nabla_{2}}{4 A_{22} A_{21}} \text {, } \\
& \nabla_{22}^{2}=\frac{\Delta^{2}-4 A_{22} \nabla_{2}}{4 A_{22}^{2}}, \nabla_{23}^{2}=\frac{\Delta^{2}-4 A_{22} \nabla_{2}}{4 A_{22} A_{23}}, \\
& \Delta_{33}=\frac{\Delta}{2 A_{33}}, \nabla_{31}^{2}=\frac{\Delta^{2}-4 A_{33} \nabla_{3}}{4 A_{33} A_{31}} \text {, } \\
& \nabla_{32}^{2}=\frac{\Delta^{2}-4 A_{33} \nabla_{3}}{4 A_{33} A_{32}} \nabla_{33}^{2}=\frac{\Delta^{2}-4 A_{33} \nabla_{3}}{4 A_{33}^{2}}, \\
& A_{11}=\operatorname{det}\left[\begin{array}{cc}
b+1 & -\alpha \\
-\beta & c+1
\end{array}\right]=(b+1)(c+1)-\alpha \beta>0 \text {, } \\
& A_{12}=-\operatorname{det}\left[\begin{array}{cc}
-\alpha & -\beta \\
-\beta & c+1
\end{array}\right]=(c+1) \alpha+\beta^{2}>0 \text {, } \\
& A_{13}=\operatorname{det}\left[\begin{array}{cc}
-\alpha & -\beta \\
b+1 & -\alpha
\end{array}\right]=\alpha^{2}+(b+1) \beta>0, \\
& A_{21}=-\operatorname{det}\left[\begin{array}{cc}
-\beta & -\alpha \\
-\alpha & c+1
\end{array}\right]=(c+1) \beta+\alpha^{2}>0 \text {, } \\
& A_{22}=\operatorname{det}\left[\begin{array}{cc}
a+1 & -\beta \\
-\alpha & c+1
\end{array}\right]=(a+1)(c+1)-\alpha \beta>0 \text {, } \\
& A_{23}=-\operatorname{det}\left[\begin{array}{cc}
a+1 & -\beta \\
-\beta & -\alpha
\end{array}\right]=(a+1) \alpha+\beta^{2}>0 \text {, } \\
& A_{31}=\operatorname{det}\left[\begin{array}{cc}
-\beta & b+1 \\
-\alpha & -\beta
\end{array}\right]=(b+1) \alpha+\beta^{2}>0 \text {, } \\
& A_{32}=-\operatorname{det}\left[\begin{array}{cc}
a+1 & -\alpha \\
-\alpha & -\beta
\end{array}\right]=(a+1) \beta+\alpha^{2}>0 \text {, } \\
& A_{33}=\operatorname{det}\left[\begin{array}{cc}
a+1 & -\alpha \\
-\beta & b+1
\end{array}\right]=(a+1)(b+1)-\alpha \beta>0 \text {, }
\end{aligned}
$$




$$
\begin{aligned}
& \Delta=\operatorname{det}\left[\begin{array}{ccc}
a+1 & -\alpha & -\beta \\
-\beta & b+1 & -\alpha \\
-\alpha & -\beta & c+1
\end{array}\right], \nabla_{1} \\
& =\operatorname{det}\left[\begin{array}{ccc}
a & -\alpha & -\beta \\
b & b+1 & -\alpha \\
c & -\beta & c+1
\end{array}\right], \\
& \nabla_{2}=\operatorname{det}\left[\begin{array}{ccc}
a+1 & a & -\beta \\
-\beta & b & -\alpha \\
-\alpha & c & c+1
\end{array}\right], \\
& \nabla_{3}=\operatorname{det}\left[\begin{array}{ccc}
a+1 & -\alpha & a \\
-\beta & b+1 & b \\
-\alpha & -\beta & c
\end{array}\right] .
\end{aligned}
$$

To determine the stability of a positive equilib riu m point of (2.1), we will use the direct method of Lyapunov:

\subsection{Direct Method of Lyapunov}

Next let us consider the local stability of a positive equilibrium point $E=\left(x^{*}, y^{*}, z^{*}\right) \in \Omega \subset R_{+}^{3}$, where $\Omega$ is a neighborhood of $E$ to be determined. Based on the "direct method" of Lyapunov, we construct a continuous function

$$
\begin{aligned}
& V(x, y, z)=\delta_{1}\left[x-x^{*}-x^{*} \ln \ln \left(\frac{x}{x^{*}}\right)\right]+\delta_{2}\left[y-y^{*}-y^{*} \ln \ln \left(\frac{y}{y^{*}}\right)\right]+ \\
& \delta_{3}\left[z-z^{*}-z^{*} \ln \ln \left(\frac{z}{z^{*}}\right)\right]
\end{aligned}
$$

where $\delta_{i}(\mathrm{i}=1,2,3)$ are positive constant numbers which are yet unspecified, satisfying the following properties:

(a) $V(E)=0$,

(b) $V(x, y, z)>0$ for $\Omega \backslash\{E\}$, that is, the equilibrium point $E$ is an isolated minimum of $V$. In fact,

$$
\begin{gathered}
V_{x}(E)=V_{y}(E)=V_{z}(E)=0, \\
V_{x x}(E)=\delta_{1} \frac{x^{*}}{x^{2}}>0 ; \operatorname{det}\left[\begin{array}{cc}
V_{x x} & V_{x y} \\
V_{y x} & V_{y y}
\end{array}\right] \\
=\delta_{1} \delta_{2} \frac{1}{x^{*} y^{*}}>0 ; \\
\operatorname{det}\left[\begin{array}{lll}
V_{x x} & V_{x y} & V_{x z} \\
V_{y x} & V_{y y} & V_{y z} \\
V_{z x} & V_{z y} & V_{z z}
\end{array}\right]=\delta_{1} \delta_{2} \delta_{3} \frac{1}{x^{*} y^{*} z^{*}}>0
\end{gathered}
$$

where the partial derivatives are calculated at $E$.

(c) The function $V$ is continuously differentiable on the neighborhood $\Omega \backslash\{E\}$, and, on this set, $V^{\prime}(x, y, z)<0$. Here,

$$
\begin{aligned}
& V^{\prime}(x(t), y(t), z(t))=\delta_{1}\left(x-x^{*}\right)[(1-x)(x-a)-\alpha y-\beta z]+ \\
& \delta_{2}\left(y-y^{*}\right)[(1-y)(y-b)-\beta x-\alpha z]+ \\
& \delta_{3}\left(z-z^{*}\right)[(1-z)(z-c)-\alpha x-\beta y .
\end{aligned}
$$

Since, $E=\left(x^{*}, y^{*}, z^{*}\right)$ is a positive equilibrium point of $\operatorname{system}(2.1), V^{\prime}$ satisfies

$$
\begin{aligned}
& V^{\prime}(x(t), y(t), z(t))= \\
& \delta_{1}\left[-\left(x-x^{*}\right)^{3}+\left(-2 x^{*}+a+1\right)\left(x-x^{*}\right)^{2}-\right. \\
& \left.\alpha\left(x-x^{*}\right)\left(y-y^{*}\right)-\beta\left(x-x^{*}\right)\left(z-z^{*}\right)\right]+ \\
& \delta_{2}\left[-\left(y-y^{*}\right)^{3}+\left(-2 y^{*}+b+1\right)\left(y-y^{*}\right)^{2}-\right. \\
& \left.\beta\left(x-x^{*}\right)\left(y-y^{*}\right)-\alpha\left(y-y^{*}\right)\left(z-z^{*}\right)\right]+ \\
& \delta_{3}\left[-\left(z-z^{*}\right)^{3}+\left(-2 z^{*}+c+1\right)\left(z-z^{*}\right)^{2}-\right. \\
& \left.\alpha\left(x-x^{*}\right)\left(z-z^{*}\right)-\beta\left(y-y^{*}\right)\left(z-z^{*}\right)\right]- \\
& \left.\alpha\left(x-x^{*}\right)\left(z-z^{*}\right)-\beta\left(y-y^{*}\right)\left(z-z^{*}\right)\right]
\end{aligned}
$$

If we prove that $E=\left(x^{*}, y^{*}, z^{*}\right)$ is an isolated maximum of $V^{\prime}(x, y, z)=L(x, y, z)$, then (c) follows easily, that is:

(c1) We note that $E$ is a critical point of the function $L(x, y, z)$, that is

$$
\begin{aligned}
& L_{x}(x, y, z)=-3 \delta_{1}\left(x-x^{*}\right)^{2}+ \\
& 2 \delta_{1} \rho_{1}\left(x-x^{*}\right)-\left(\alpha \delta_{1}+\beta \delta_{2}\right)\left(y-y^{*}\right)- \\
& \left(\beta \delta_{1}+\alpha \delta_{3}\right)\left(z-z^{*}\right) L_{y}(x, y, z) \\
& =-3 \delta_{2}\left(y-y^{*}\right)^{2}+2 \delta_{2} \rho_{2}\left(y-y^{*}\right)- \\
& \left(\alpha \delta_{1}+\beta \delta_{2}\right)\left(x-x^{*}\right)-\left(\alpha \delta_{2}+\beta \delta_{3}\right)\left(z-z^{*}\right) \\
& L_{z}(x, y, z)=-3 \delta_{3}(z-z)^{2}+2 \delta_{3} \rho_{3}\left(z-z^{*}\right)- \\
& \left(\beta \delta_{1}+\alpha \delta_{3}\right)\left(x-x^{*}\right)-\left(\alpha \delta_{2}+\beta \delta_{3}\right)\left(y-y^{*}\right)
\end{aligned}
$$

implies $L_{x}(E)=L_{y}(E)=L_{z}(E)=0$.

(c2) The equilibrium point $E$ is a maximum point of $L(x, y, z)<=>$

$\left(i^{\prime}\right) L_{x x}(E)<0 ;\left(i i^{\prime}\right) H_{1}(E)>0 ;\left(i i i^{\prime}\right) H(E)<0(3.10)$

Here

$$
\begin{gathered}
L_{x x}(E)=2 \delta_{1} \rho_{1} ; \\
H_{1}(E)=\left(\begin{array}{cc}
L_{x x}(E) & L_{x y}(E) \\
L_{y x}(E) & L_{y y}(E)
\end{array}\right)=\left(\begin{array}{cc}
2 \delta_{1} \rho_{1} & -\alpha \delta_{1}-\beta \delta_{2} \\
-\alpha \delta_{1}-\beta \delta_{2} & 2 \delta_{2} \rho_{2}
\end{array}\right) \\
H(E)=\left[\begin{array}{ccc}
L_{x x}(E) & L_{x y}(E) & L_{x z}(E) \\
L_{x y}(E) & L_{y y}(E) & L_{y z}(E) \\
L_{x z}(E) & L_{y z}(E) & L_{z z}(E)
\end{array}\right] \\
=\left[\begin{array}{ccc}
2 \delta_{1} \rho_{1} & -\alpha \delta_{1}-\beta \delta_{2} & -\beta \delta_{1}-\alpha \delta_{3} \\
-\alpha \delta_{1}-\beta \delta_{2} & 2 \delta_{2} \rho_{2} & -\alpha \delta_{2}-\beta \delta_{3} \\
-\beta \delta_{1}-\alpha \delta_{3} & -\alpha \delta_{2}-\beta \delta_{3} & 2 \delta_{3} \rho_{3}
\end{array}\right]
\end{gathered}
$$

Letting $\delta_{1}=\delta_{2}=1 / 2$, we can rewrite (3.10) as

$$
\begin{aligned}
& \rho_{1}<0 ; \rho_{1} \rho_{2}-\tau^{2}>0 ; \\
& \rho_{1} \rho_{2} \rho_{3}-\left(\rho_{1}+\rho_{2}+\rho_{3}\right) \tau^{2}+2 \tau^{3}<0, \\
& \tau=-\frac{(\alpha+\beta)}{2} .
\end{aligned}
$$

Thus, $E=\left(x^{*}, y^{*}, z^{*}\right)$ is a isolated maximum of $V^{\prime}(x, y, z)$, i.e., there is a neighborhood $\Omega$ of $E$ such that 
$V^{\prime}(x, y, z)<0$, on this set. The pertinent result, which we prove, is the following (see Fig.3.3):.

Proposition 3.5. Consider the Lyapunov function (3.8) defined in the neighborhood $\Omega \subset R_{+}^{3}$ of a positive equilibrium point $E$ of the competitive system (2.1). If (3.11) occurs, then $E=\left(x^{*}, y^{*}, z^{*}\right)$ is locally asymptotically stable.

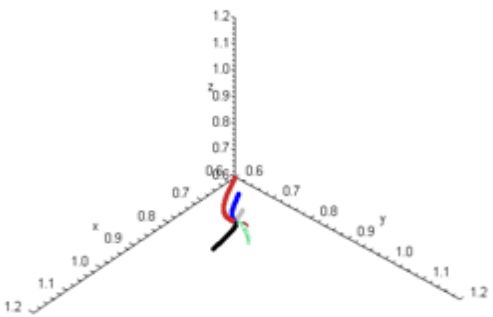

Figure 3.3. Each graph depicts a three-dimensional $x, y, z$ population evolution in the state space for system (2.1). The initial conditions are $(0,0,0),(0.9,0.9,0.9),(0.8,0.8,0.8),(0.7,0.7,0.7),(0.6,0.6,0.6),(1.0,0.9,0.9) . T h$ e equilibrium point $E_{+++}^{5}$ is locally asymptotically stable. Here $a=$ $0.1, b=0.2, c=0.15, \alpha=\frac{1}{16}, \beta=\frac{1}{25}$

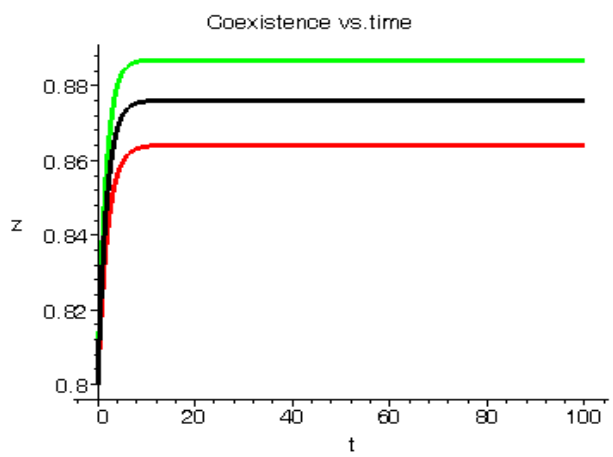

Figure 3.4. Each graph depicts one-dimensional $x, y, z$ population changes with respect to time for system (2.1). Each trajectory starts at a point $(0.8,0.8,0.8)$ near the equilibrium $E_{+++}^{5}$ locally asymptotically stable: green $-x$, red $-y$, black $-z$. Here $a=0.1, b=0.2, c=0.15, \alpha=\frac{1}{16}, \beta=$ $\frac{1}{25}$

\section{Numerical example}

By using the software of MAPLE 10, a numerical example has been provided to illustrate the behavior of the system (2.1) for a biologically reasonable range of parameters. Choosing the following set of values for the parameters in (2.1):

$$
a=0.1, b=0.2, c=0.15, \alpha=1 / 16, \beta=1 / 25,
$$

we find that the inequalities given by (3.11) hold for a unique positive equilibrium point and we observe that there are 27 equilibriu m points given by

$$
\begin{gathered}
E_{000}=(0,0,0), E_{a 00}=(0.1,0,0), E_{100}=(1,0,0), \\
E_{0 b 0}=(0,0.2,0), \\
E_{010}=(0,1,0), E_{00 c}=(0,0,0.15), E_{001}=(0,0,1), \\
E_{++0}^{1}=(0.1145238077,0.2057677744,0), \\
E_{++0}^{2}=(0.982105439,0.2525582316,0),
\end{gathered}
$$

$E_{++0}^{3}=(0.9282751363,0.9505267387,0)$, $E_{++0}^{4}=(0.1750956168,0.9911472553,0)$, $E_{+0+}^{1}=(0.1145238077,0,0.1070755147)$, $E_{+0+}^{2}=(0.9896422935,0,0.2303663443)$, $E_{+0+}^{3}=(0.9569355368,0,0.9225867215)$, $E_{+0+}^{4}=(0.1463466549,0,0.9890994253)$, $E_{0++}^{1}=(0,0.2127122137,0.1601307297)$, $E_{0++}^{2}=(0,0.2863822446,0.98863024559)$, $E_{0++}^{3}=(0,0.9167794511,0.9544124694)$, $E_{0++}^{4}=(0,0.9841260905,0.1991543450)$, $E_{+++}^{1}=(0.1234346734,0.2199416141,0.1698917819)$, $E_{+++}^{2}=(0.9689920329,0.2745078943,0.2447233248)$, $E_{+++}^{3}=(0.1690910317,0.2959630865,0.9727666239)$, $E_{+++}^{4}=(0.9306479263,0.3426568704,0.9047775459)$, $E_{+++}^{5}=(0.8868214327,0.8641473928,0.8760527607)$, $E_{+++}^{6}=(0.2205803755,0.9043799491,0.9364741050)$, $E_{+++}^{7}=(0.9152759525,0.9253052261,0.2810475482)$, $E_{+++}^{8}=(0.1851565751,0.9730979666,0.2142663096)$. The $y$-coordinate of the positive equilibrium points $E_{+++}^{i}(\mathrm{i}=1 \ldots 8)$ are roots of $(3.5)$, that is $F(y)=0$, where

$$
\begin{aligned}
& F(y)=-\left(\frac{65536}{390625}\right) y^{8}+0.8053063680 y^{7}- \\
& 1.622650388 y^{6}+1.78271132 y^{5}-1.162155201 y^{4}+ \\
& 0.4589826936 y^{3}-0.1073327879 y^{2}+0.01363187734 y- \\
& 0.0007228687716
\end{aligned}
$$

In the equilibrium point $E_{+++}^{5}$, the characteristic equation (4.11) of $J\left(E_{+++}^{5}\right)$ reduces to $p(\lambda)=\lambda^{3}+1.581401696 \lambda^{2}+0.8228981025 \lambda+$ 0.1410366097 , with roots $-0.6392646908,-0.5068784588,-0.4352585464$ This implies that $E_{+++}^{5}$ is a locally asymptotically stable equilibrium point. Here, we observe that the equilibrium points $E_{+++}^{i}(i \neq 5)$ are unstable.

In the absence of a competitor, we have: (a) $E_{++0}^{3}$ is a locally asymptotically stable equilibrium point, with eigenvalues $-0.7346114284, \quad-0.6340454548$ and 0.2460382656 . The equilibrium points $E_{++0}^{j}(j \neq 3)$ are unstable. (b) $E_{+0+}^{3}$ is a locally asymptotically stable equilibrium point, with eigenvalues -0.7933441170, $0.6268358235,-0.2959390916$. The equilibrium points $E_{+0+}^{j}(j \neq 3)$ are unstable. (c) $E_{0++}^{3}$ is a locally asymptotically stable equilib riu m point, with eigenvalues $0.7381379376,-0.5669278285, \quad-0.1954752145$. The equilibrium points $E_{0++}^{j}(j \neq 3)$ are unstable. 
In the absence of two competitors, we have: (d) $E_{100}$ is a locally asymptotically stable equilibrium point, with eigenvalues $-0.9,-0.24,-0.2125$. The equilibrium $E_{a 00}=(0.1,0,0)$ is unstable. (e) $E_{010}$ is a locally asymptotically stable equilibrium point, with eigenvalues $-0.8,-0.14,-0.2625$. The equilibrium $E_{0 b 0}=(0,0.2,0)$ is unstable. (f) $E_{001}$ is a locally asymptotically stable equilibrium point, with eigenvalues $-0.85,-0.241625,-0.19$. The equilibrium $E_{00 c}=$ $(0,0,0.15)$ is unstable.

Clearly $E_{000}=(0,0,0)$ is a locally asymptotically stable equilibrium point, with eigenvalues $-0.1,-0.2,-0.15$, respectively.

The $x$-coordinate of the equilibrium points $E_{++0}^{i}$ $(\mathrm{i}=1 \ldots 4)$ are roots of $(3.3) p(x)=256 x^{4}-563.2 x^{3}+$ $380.16 x^{2}-77.4 x+4.68$. Intersection between two ellipses:

$$
\begin{gathered}
E_{1}:(0.5489583333+0.436965199 \cos \cos (t), \\
1.914685571 \sin \sin (t)) \\
E_{2}:(2.064590892 \cos (t), 0.5988636364+ \\
0.3937020336 \sin (t)) ; \mathrm{t}=0.2 \pi
\end{gathered}
$$

The $x$-coordinate of the equilibrium points. $E_{+0+}^{i}$ $(\mathrm{i}=1 \ldots 4)$ are roots of $q(x)=625 x^{4}-1375 x^{3}+910 x^{2}-$ 169.0625x +9.275 . Intersection between two ellipses:

$$
E_{3}:(0.5489130435+0.4428184030 \cos (t),
$$$$
2.374349167 \sin \sin (t)) \text {; }
$$

$$
E_{4}:(1.748148381 \cos (t),
$$

$0.5738863664+0.4166985181 \sin (t)) ; t=0 \ldots .2 \pi$.

The equilibrium points are: $E_{+0+}^{1}$ is an unstable node; $E_{+0+}^{2}$ is a saddle point; $E_{+0+}^{3}$ is locally asymptotically stable; $E_{+0+}^{4}$ is a saddle point.

The $y$-coordinate of the equilibrium points $E_{0++}^{i}$ $(\mathrm{i}=1 \ldots 4)$ are roots of the polynomial: $r(y)=256 y^{4}-$ $614.4 y^{3}+489.44 y^{2}-144.92 y+14.070$. Intersection between two ellipses:

$$
\begin{gathered}
E_{5}:(0.5989130435+0.3880008501 \cos \cos (t), \\
1.664338228 \sin \sin (t)) \\
E_{6}:(2.276586272 \cos (t), 0.5739583333+ \\
0.415645885 \sin (t)) ; \mathrm{t}=0 \ldots 2 \pi .
\end{gathered}
$$

The equilibrium points are: $E_{0++}^{1}$ is an unstable node; $E_{0++}^{2}$ is a saddle point; $E_{0++}^{3}$ is locally asymptotically stable; $E_{0++}^{4}$ is a saddle point.

\section{Concluding Remarks}

In this paper, a mathematical model of competition between three populations with lower threshold sizes has been proposed and investigated. The main focus was to analyze the question of existence and stability of nonnegative equilibria. Our results show that there exist at most twenty-seven equilibrium points for the system under consideration and, by using the software of MAPLE 10, a numerical example has been provided to illustrate the behavior of the system (2.1) for a biologically reasonable range of parameters with only one positive equilibrium asymptotically stable and 7 positive unstable equilibrium points.

\section{REFERENCES}

[1] Brauer,F., Castillo-Chávez,C. "Mathematical Models in Population Biology and Epidemiology", Springer-Verlag, New York, 2001.

[2] Wang,G., Liang, X-G., Wang,F-Z., "The competitive dynamics of populations subject to an Allee effect", Ecological Modelling, vol 124, no 2,pp. 183-192,1999.

[3] Zhou,S-R., Liu,C-H., Wang,G. "The competitive dynamics of metapopulations subject to the Allee-like effect", Theoretical Population Biology, vol 65, no 1, pp.29-37,2004.

[4] Bazykin A.D. "Nonlinear Dynamics of Interacting Populations". World Scientific , Sin gapore, 1998.

[5] Freedman H.I. "Deterministic Mathematical Models in Population Ecology”. Marcel Dekker, Inc., New York, 1980.

[6] Hofbauer,J., Sigmund, K. "The Theory of Evolution and Dynamical Systems", Cambridge University Press, Cambrid ge, 1992.

[7] Freedman,H.I., Waltman, P. "Persistence in a model of three competitive populations", Mathematical Biosciences, vol 73, no 1, pp.89-101,1985.

[8] May, R.M., Leonard,W. "Nonlinear aspects of competition between three species", SIAM Journal on Applied Mathematics, vol 29,no 2,243-252,1975.

[9] Rescigno, A. "The struggle for life:II. Three competitors", Bulletin of Mathematical Biophysics, vol 30,no 2,pp.291298,1968 .

[10] Takeuchi,Y. "Global Dynamical Properties of LotkaVolterra Systems". World Scientific Publishing, Singapore, 1996.

[11] Phillipson,P.E., Schuster,P., Johnson,R.G. "An analytical study of the May-Leonard equation", SIAM Journal on Applied Mathematics, vol 45,no 4,pp.541-554,1985.

[12] Afraimovich, V.S., Hsu,S-B., Lin,H-E. "Chaotic behavior of three competing species of May-Leonard model under small periodic perturbations", International Journal of Bifurcation and Chaos. vol 11, no 2, pp.435-447, 2001.

[13] Chi,C-W., Hsu, S-B., Wu,L-I. "On the asymmetric MayLeonard model of three competing species", SIAM Journal on Applied Mathematics, vol 58, no 1,pp.211-226,1998.

[14] Schuster,P., Sigmund,K., Wolff,R. "On $\omega$-limits for competition between three species", SIAM Journal on Applied Mathematics, vol 37, no 1, pp.49-54,1979.

[15] Li,X., Wei, J.,"On the zeros of a fourth degree exponential polynomial with applications to a neural network model with delays", Chaos, Solitons \& Fractals, vol 26, no 2, pp.519526,2005 . 